\title{
SoCIAL - training cognition in schizophrenia: a pilot study
}

\author{
This article was published in the following Dove Press journal: \\ Neuropsychiatric Disease and Treatment \\ 20 July 2017 \\ Number of times this article has been viewed
}

\section{Davide Palumbo* \\ Armida Mucci* \\ Giuseppe Piegari \\ Valentina D'Alise \\ Annapaola Mazza \\ Silvana Galderisi}

Department of Psychiatry, University of Campania Luigi Vanvitelli,

Naples, Italy

*These authors contributed equally to this work
Correspondence: Davide Palumbo Department of Psychiatry, University of Campania Luigi Vanvitelli, Largo Madonna delle Grazie I, 80138 Naples, Italy

Tel +3908I 5666532

Email da.palumbo@outlook.it

\begin{abstract}
The purpose of this pilot study was to assess the efficacy of a new social cognition (SC) remediation intervention, the Social Cognition Individualized Activities Lab (SoCIAL), for subjects with schizophrenia. The training includes a module for emotion recognition and one for theory of mind. A comparison with a validated cognitive remediation intervention, the Social Skills And Neurocognitive Individualized Training (SSANIT), was conducted to verify the efficacy of the SoCIAL in improving SC. Ten stabilized patients with schizophrenia accepted to participate. Five patients were randomized to SoCIAL and five to SSANIT. The SoCIAL intervention includes individual sessions of neurocognitive individualized training (NIT) and group sessions of SC training. SSANIT includes individual sessions of NIT and group sessions of social skills individualized training. The interventions were matched for the overall treatment duration (20 weeks) and for the frequency of the sessions (two times a week, one for SoCIAL or social skills individualized training and one for NIT, with a duration of 80 minutes for each session). Results showed a significant treatment effect (effect size: Cohen's d 0.32) on the primary outcome; in fact, only the SoCIAL intervention improved theory of mind. Patients receiving the SoCIAL intervention also showed an improvement of avolition. These preliminary findings support further development of the SoCIAL and suggest that cognitive remediation should include an SC module.
\end{abstract}

Keywords: cognitive remediation, psychiatric rehabilitation, negative symptoms, avolition, MATRICS consensus cognitive battery, MCCB

\section{Introduction}

Impairment in several domains of cognition has been reported in patients with schizophrenia. ${ }^{1-5}$ Cognitive dysfunctions are associated with poor functional outcome ${ }^{6,7}$ and quality of life ${ }^{8}$ in these patients. Evidence has been provided that cognitive deficits have a greater impact on functional outcome than positive or negative symptoms. ${ }^{9,10}$

For a specific domain of cognition, that is, social cognition (SC), there is a growing consensus among researchers about its impact on functional outcome, beyond other cognitive domains, and its key role in mediating the relationship between patients' real-life functioning and other cognitive domains (the so-called neurocognitive functions, such as attention, memory, executive control and processing speed). ${ }^{9,11}$

$\mathrm{SC}$ involves mental operations underlying social interactions, such as perceiving, interpreting and generating responses to intentions, dispositions and behaviors of others. ${ }^{12,13}$

The vast majority of research on SC in schizophrenia has focused on four generally accepted domains of SC: the interrelated abilities of processing facial emotion (facial affect recognition) and interpreting and responding to social cues, such as body language or voice intonation (social perception), the ability to understand that other 
people may have different mental states than oneself(Theory of Mind [ToM]) and the ability to make suitable attributions of the causes of events (attributional style). ${ }^{12,13}$ Impairment in each of these domains has been shown to have a significant impact on functional outcome in patients with schizophrenia and to explain variance in functional outcome above and beyond neurocognition..$^{9,11,14-16}$

Despite the key role of cognitive impairments in schizophrenia, to date, drug treatments have only a marginal impact on neurocognitive and SC deficits; ${ }^{17,18}$ therefore, there is an urgent need of new treatment strategies for patients with schizophrenia to achieve clinically relevant enhancement in these functional domains.

For these reasons, in the last decades, several cognitive remediation interventions have been implemented to improve cognition, mainly focusing on neurocognitive functions. ${ }^{19,20}$ Rehabilitation interventions specifically targeting SC are still at an early stage of development, but initial results are encouraging. ${ }^{13,21,22}$

Multiple approaches have been taken in developing these latter programs that vary across several dimensions: some of them include a training for a single domain of SC and are generally referred to as "targeted" interventions, ${ }^{22}$ while others combine multiple domains to create more complex programs or "broad-based" interventions. ${ }^{21,23}$ Some interventions are brief, including one or two sessions, while others are more continuous, lasting several weeks or months. ${ }^{24-26}$

A meta-analysis on social cognitive training showed that the results are consistent with the hypothesis that social cognitive training programs produce improvement of facial affect recognition in the moderate-large range, while producing a smaller but significant effect on ToM. ${ }^{27}$ Findings regarding other SC domains are more disappointing. ${ }^{28}$ Finally, the results of two meta-analyse ${ }^{20,25}$ highlighted that the most significant effects on real-life functioning can be demonstrated when cognitive training is administered together with other psychosocial rehabilitation programs (such as social skills training, supported employment, illness self-management) and when a strategy coaching approach based on learning strategies is adopted. ${ }^{28}$

In line with these evidences, our research group demonstrated the effectiveness of a structured and individualized rehabilitative program, the Social Skills And Neurocognitive Individualized Training (SSANIT), including a computerized neurocognitive individualized training (NIT) and a social skills individualized training (SSIT), in patients affected by schizophrenia or schizoaffective disorder, in improving daily functioning and quality of life, ${ }^{29}$ and reported that although both NIT and SSIT have a favorable impact on domains of functional outcome, only NIT improves cognitive functioning. ${ }^{30}$

In this pilot study, we describe a newly developed structured rehabilitation intervention, the Social Cognition Individualized Activities Lab (SoCIAL), designed for patients with schizophrenia or schizoaffective disorder, specifically aimed to improve SC. The SoCIAL includes a targeted training of two dimensions of SC: emotion recognition (ER) and ToM. In this pilot study, SoCIAL was integrated with NIT and compared to the previously validated intervention, SSANIT.

\section{Patients and methods}

This pilot study had a controlled design including randomization to treatment groups, blind assessments and stable pharmacologic treatment.

\section{Subjects}

Inclusion criteria for entering the pilot study were: 1) age between 18 and 50 years; 2) clinical diagnosis of either schizophrenia or schizoaffective disorder, according to the Diagnostic and Statistical Manual of Mental Disorders Fourth Edition criteria, in a stable phase of the illness; 3 ) illness duration of $<10$ years; 4) absence of significant changes in drug treatment during the 3 months prior to recruitment and 5) minimum of 5 years of schooling. Exclusion criteria were: 1) presence of diseases involving disability and 2) habitual use of alcohol or drugs in the last 6 months. Patients with a diagnosis of schizophrenia or schizoaffective disorder, among those consecutively visited within 1 month (December 2013) at the outpatient unit for schizophrenia spectrum disorders of our University Department of Mental Health, were asked to participate in this pilot study. The study was approved by the Ethics Committee of the Azienda Ospedaliera of the University of Campania "Luigi Vanvitelli". Ten patients agreed to participate in this study and signed written informed consent; five patients were randomized to SoCIAL (three females and two males) and five (three females and two males) to SSANIT. A patient from the latter group moved to another city before completing 1 month of treatment and was not included in the analyses. Details of the reasons for patients' exclusion and number of completers are reported in Figure 1.

\section{Implementation of the training program}

The study included the following phases: 1) implementation of ecological materials for SoCIAL (photographs and videos 


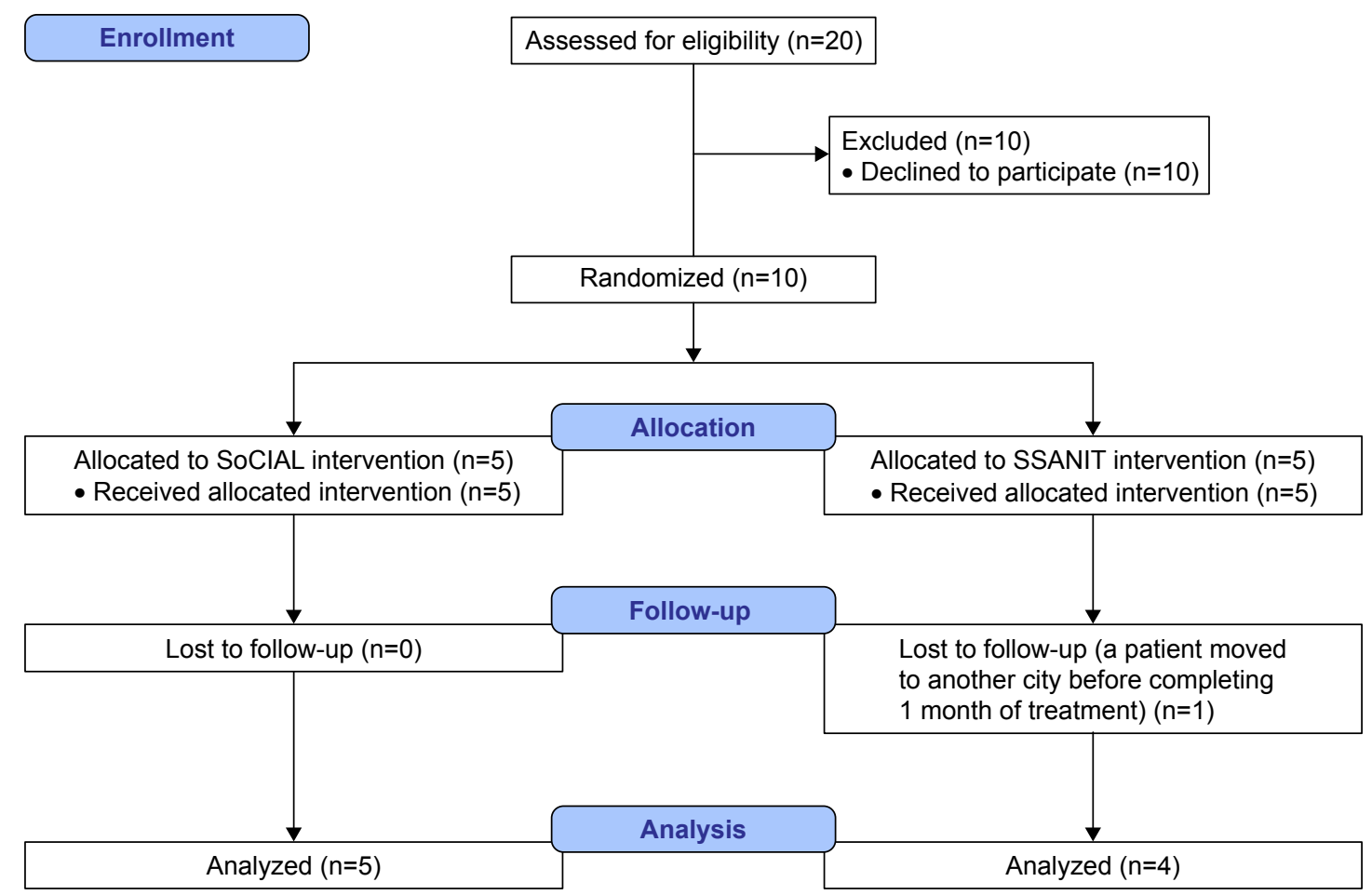

Figure I Flow diagram of the study.

Abbreviations: SoCIAL, Social Cognition Individualized Activities Lab; SSANIT, Social Skills And Neurocognitive Individualized Training.

involving professional actors, sound engineers, scriptwriters and a director); 2) referrer training for both the intervention programs before recruitment of the subjects; 3) baseline assessment of psychopathology, neurocognitive functions, functional capacity, perceived quality of life and SC of patients enrolled in the study; 4) 20-week active intervention (SSANIT or SoCIAL integrated with NIT) and 5) posttreatment evaluation of psychopathology, neurocognitive functions, functional capacity, perceived quality of life, SC and patients' level of satisfaction.

\section{Photo and video materials used in the SoCIAL}

Ecological materials were developed with the involvement of professional actors, scriptwriters, sound engineers and a director. Psychiatrists and psychologists specialized in the field of psychiatric rehabilitation supervised the development of the training materials. The module "Emotion Recognition" comprises 10 videos in which a professional actor portrays one of five basic emotional states (happy, sad, fearful, disgusted and angry). In each vignette, the actor is engaged in an interaction with one or more participants using a script that is ambiguous and can therefore be interpreted in a number of ways.

The module "Theory of Mind" comprises 15 videoclips of social interactions. The videoclips show trained actors in dynamic displays of emotions, as well as in conversational interactions with others, requiring the integration of multiple changing cues (face expression, prosody, gesture and context) to identify the emotions, beliefs and intentions of target characters within the social context. The videos thus provide an ecologically valid measure of SC that assesses several domains of SC (ER and ToM) showing six different social interactions comprising sarcasm, disappointment, hostility, misunderstanding, deceit and sincere.

The materials have been designed, since the beginning, for a training program, in order to ensure a greater ecological validity. Moreover, the use of simple and everyday contexts such as family, friendship and work, in our opinion, brings the training closer to the real life of patients with schizophrenia, and we assume that this will increase the effectiveness of video stimuli for SoCIAL.

\section{Training of the personnel and intervention fidelity assurance}

Six social workers, two psychologists and four psychiatric rehabilitation technicians (PRTs) of the Department of Psychiatry of the Second University of Naples, were trained by an expert psychiatrist in rehabilitation programs of the same center (two PRTs for NIT, one psychologist and one PRT both for SoCIAL and the SSIT component of SSANIT). 
During the 3-month training, these personnel attended ten 2-hour sessions and became familiar with the theory and practice of NIT, SoCIAL and SSIT. After the training, the personnel had to be able to design the interventions, to supervise patients for the NIT program and to be trainer or co-trainer for the SoCIAL and SSIT groups. Intervention fidelity to NIT, SoCIAL and SSIT was assessed by means of supervisory meetings held once a month, in which the trainer supervised the activity of the trained personnel. For SoCIAL and SSIT groups, the supervision was carried out by addressing patients' individual difficulties: this represented the starting point to discuss strategies used in coaching, providing corrective/positive feedback and assigning homework, as well as managing difficulties encountered during the sessions; similarly, for NIT, the supervision was carried out by evaluating the computer outputs relevant to progression on each module during the session, with subsequent discussion of strategies used in selecting the level of difficulty as well as in giving positive or corrective feedback.

\section{Assessment of clients' satisfaction, psychopathology, neurocognitive functions, SC, functional capacity and real- life functioning}

At the end of the SoCIAL training, we used the Client Satisfaction Questionnaire (CSQ-8), ${ }^{31}$ an eight-item version, to evaluate patient satisfaction. The sum of the responses to the CSQ-8 ranges from 8 to 32, with higher scores indicating greater satisfaction. Psychopathological evaluation was carried out by means of the Positive and Negative Syndrome Scale (PANSS). ${ }^{47}$ Ratings on PANSS items were summed to calculate two dimensions of schizophrenia symptomatology: positive symptoms and disorganization, according to the method proposed by Wallwork et al. ${ }^{32}$

Negative symptoms were assessed using the Brief Negative Symptom Scale, composed of 13 items, rated from 0 (normal) to 6 (most impaired), and five negative symptom domains: anhedonia, asociality, avolition, blunted affect and alogia. The Italian version of the scale was validated in the wider study of the Italian Network for Research on Psychoses activities. ${ }^{33}$ In line with previous research, ${ }^{9}$ domains evaluated by the scale loaded on two factors: "avolition", which includes anhedonia, asociality and avolition, and "expressive deficit", which evaluates blunted affect and alogia. ${ }^{34}$

Neurocognitive functions were rated using the MATRICS consensus cognitive battery (MCCB) that includes tests for the assessment of different cognitive domains.
We evaluated processing speed, attention/vigilance, working memory, visual learning, verbal learning and reasoning and problem solving. ${ }^{35,36}$

The assessment of SC included the Facial Emotion Identification Test (FEIT), ${ }^{37}$ which examines facial affect recognition, and the Awareness of Social Inference Test (TASIT), ${ }^{38}$ which is a ToM test consisting of seven scales (positive emotions, negative emotions, sincere, simple sarcasm, paradoxical sarcasm, sarcasm enriched and lie), organized into three sections: ER, social inference (minimal) and social inference (enriched). The manual of the TASIT was translated into Italian by a psychiatrist of the Department of Psychiatry of the University of Naples SUN, who gained experience in the use of the English version of the instrument during his stage at the Department of Psychiatry and Biobehavioral Sciences at the University of California, Los Angeles, as part of his $\mathrm{PhD}$ course. The videotaped vignettes of the TASIT were dubbed in Italian at the Fono Roma Studios (http://www. fonoroma.com), a prestigious society in the field of film industry. As to the FEIT, the adaptation of the Italian version required the translations of the six emotions reported on the screen above the stimuli.

Functional capacity was evaluated by the short version of the University of California San Diego Performance-based Skills Assessment Brief, ${ }^{39}$ a performance-based instrument that assesses "financial skills" (counting money and paying bills) and "communication skills" (to dial a telephone number for emergency or reschedule an appointment by telephone). The total score, starting from 0 (poorest functional capacity) to 100 (best functional capacity), was used in statistical analyses.

Real-life functioning was assessed using the Specific Level of Functioning Scale (SLOF), a hybrid instrument that examines several aspects of functioning. It is based on key caregiver's judgment on behavior and functioning of patients and consists of 43 items including six domains: physical efficiency, skills in self-care, interpersonal relationships, social acceptability, community activities (eg, shopping, using public transportation) and working abilities. For outpatients living in the community, a ceiling effect can be expected for self-care and social acceptability; thus, according to Sabbag et $\mathrm{al},{ }^{40}$ three SLOF subscales can be used in this population: interpersonal relationship, community activities and working abilities. Higher scores correspond to better functioning. In our study, the key relative was interviewed, as usually this is the individual most frequently and closely in contact with the patient in the Italian context. The Italian version of the scale was validated as part of the Italian Network for Research on Psychoses activities. ${ }^{6}$ 


\section{Interventions}

The two programs were matched for the overall treatment duration (20 weeks), as well as for the frequency (two times a week, one for SoCIAL or SSIT and one for NIT) and duration of the sessions ( 80 minutes). Both interventions included individual sessions of NIT; the SoCIAL program included group sessions of ER (first 10 sessions) and ToM (next 10 sessions), while the SSANIT program included group sessions of SSIT (communication skills in the first 10 sessions and assertive skills in the last 10 sessions).

\section{Neurocognitive individualized training}

NIT is based on a computer-assisted cognitive rehabilitation program, RehaCom, developed by the HASOMED GmbH (Inc., Ltd) in Magdeburg, Germany. ${ }^{29,30}$ The patient can work with RehaCom on a personal computer, using a special keyboard with simple, large and clear keys. All training procedures can be adapted to the individual needs and the trainer can decide on the basis of his/her judgment to reduce or increase the level of difficulty for each module.

The program provides different difficulty levels: when the subject successfully passes a level, the program will automatically switch to more complex level; if the subject finds difficulty in an exercise, the program passes immediately to the simplest level. The threshold for passage from one level of difficulty to another is set by the trainer, based on the cognitive abilities of the subject. Therefore, the training never appears too complex or too simple for the trainee. The motivation of the person being trained is supported by closeto-reality practices and positive feedback. The data obtained during the training are registered on the computer that provides both an immediate feedback on task performance and a post-session feedback for all the modules informing the trainer and the patient of the session outcome, that is, the level of difficulty successfully performed by the subject.

On the basis of the results displayed on the computer, the trainer provides the patient with positive and/or corrective feedback and strategy coaching or compensatory skills training. The following training modules were used: "Attention and Concentration", "Verbal Memory", "Memory for faces", "Shopping" and "Day planning". A description of the modules can be found elsewhere. ${ }^{29,30}$ Subjects attended two $1 \mathrm{~h}$ individual sessions per week for 20 weeks $(40 \mathrm{~h})$.

\section{Social skills individualized training}

The SSIT component of the SSANIT is aimed at improving individual assertiveness and conversation skills. ${ }^{29,30,41}$ SSIT was carried out by a psychologist in the role of a conductor and by a PRT in the role of a co-conductor. Patients attended weekly 80 -minute sessions for 20 weeks.

Training individual assertiveness promotes a positive attitude toward oneself and the others ${ }^{41}$ by improving the ability to express emotions, needs and personal opinions clearly and effectively without excessive anxiety and/or aggression. The target of this section of the training was on the ability to recognize and articulate unpleasant and positive feelings, apologize and share their fears. Dialogue skills involve the subjects' ability to listen to others, start a conversation, hold a conversation (asking questions and expressing their feelings and opinions) and finish a conversation. Individualization of SSIT component of the SSANIT uses the same strategies described by Bellack et al ${ }^{42}$ modeling, feedback, behavioral rehearsal with coaching and corrective/positive feedback, problem-solving exercises, in vivo exercises and homework assignments, but was more devised on the individual need of the subjects and, together with the training of behavioral component, had as its target the training of certain key aspects of the SC (recognition and expression of emotions). Real-life aspects of the patient are modeled into SSIT; the subjects were asked to report some special episodes involving them recently at home or in other social contexts, so as to set the training on them and on the specific social skill deficit. To promote generalization from training to real life, the patients' attempts to practice the skills outside the training group were carefully reviewed in subsequent sessions, positive feedbacks were provided and any difficulties perceived by the person were discussed with the group.

\section{Social Cognition Individualized Activities Lab}

SoCIAL was carried out by a psychologist in the role of conductor and by a PRT in the role of co-conductor. As already mentioned, the training is divided into two modules comprising two different areas of SC:

- ER module trains the ability to discriminate emotions and consists of 10 sessions, 2 sessions for each basic emotion: fear, anger, surprise, sadness and joy. Educational materials (photos and videos) are used to analyze prototypical elements of each emotion (facial micro expressions, prosody, gestures and so on). In particular, the ER module is divided into two phases:

1. Emotion prototypical elements: During this phase, patients are provided with didactic information on how to identify facial expressions of basic emotions. For this purpose, several pictures depicting emotional expressions in static images of faces have been used. In addition, patients are trained to recognize prosodic 
features (voice) and dynamic features (physical, eg, gestures, posture) of emotions using clips depicting emotionally connoted everyday situations.

2. Role-play: the role-play is meant to train patients to "live" decode the emotion expressed by the actor (co-conductor) with whom they will act the scene. Besides ER, patients are also asked to modulate an appropriate response to the emotion elicited by the co-conductor during the scene.

- ToM module trains the ability to discriminate emotional expressions in social contexts and the ability to attribute mental states to others (to understand how they feel and what they think in real-life situations). The ToM training includes 10 sessions that illustrate, using 15 videos, the following social interaction: sarcasm, disappointment, hostility, misunderstanding, deceit and sincere. The patient is required to argue about the thoughts, feelings (including subtle emotions or misunderstandings), intentions and meaning of the actors of the videos, based upon their facial expression, tone of voice and gesture.

The ToM Module is divided into two phases:

1. Identifying social and contextual cues to understand the mental states of others: patients acquire skills to identify social cues which are useful for a proper understanding of the mental states of others. In particular, three videos (with identical or similar scripts) are shown. Videos 1 and 2 train the ability to interpret conversational remarks meant nonliterally (ie, disappointment, sarcasm, deceit and so on). Video 3 trains the ability to interpret conversational remarks meant literally (ie, sincere), so that patients are able to focus their attention mainly on contextual aspects, gestures and all the social cues that allow a proper understanding of mental states of others.

2. Role-play: During the role-play, patients are trained in recognizing "online" the mental state of the actor (co-conductor) with whom they act the scene. Besides recognition of the mental states of others, patients are also asked to modulate an appropriate response to the intentions of the actor (co-conductor) during the scene.

\section{Statistical data analysis}

To evaluate participants' satisfaction, the sum of all items of the CSQ has been calculated.

The $t$-test for independent samples was used to test group differences in demographic and clinical variables at baseline.

Scores on the indices of SC were calculated by adding the number of correct answers for both FEIT (facial affect recognition) and for each section of the TASIT (ToM). Furthermore, a TASIT global score was obtained by adding the three sections of the instrument in line with the previous studies. ${ }^{9,38}$

A neurocognitive composite score was calculated by using the MCCB Computer Scoring Program through a correction of the raw scores by age, gender and education.

As to psychopathological indices, the PANSS scores for positive dimension (sum of the scores on delusions, hallucinatory behavior, grandiosity, unusual thought content) and disorganization (sum of the scores on conceptual disorganization, poor attention and difficulty in abstraction) were calculated according to Wallwork et al, ${ }^{32}$ while the negative dimensions, "Avolition" (anhedonia, asociality and avolition) and "Expressive deficit" (blunted affect and alogia), were calculated from Brief Negative Symptom Scale scores, according to previously published factor analyses. ${ }^{33,34}$

According to Sabbag et al, ${ }^{40}$ we have used three SLOF subscales (interpersonal relationship, community activities and work skills) to assess the SLOF global score in our sample.

\section{Primary outcome measures}

We compared post-pre changes in SC between the two interventions (SoCIAL and SSANIT) with an analysis of covariance (ANCOVA), entering the change scores on each test (FEIT total score and TASIT global score) as the dependent variable, treatment as the fixed group effect and pretreatment neurocognitive composite score as the covariate. If the group effect was significant, follow-up univariate test ( $t$-test for dependent samples) was carried out in each group to verify whether the effect was due to improvement in the SOCIAL group or deterioration in the SSANIT group.

\section{Secondary outcome measures}

The effect of treatment on neurocognitive composite scores was analyzed using an ANCOVA with the composite change scores as the dependent variable, treatment as the fixed group effect and pretreatment neurocognitive composite score as the covariate.

Functional outcome measures were compared between groups using an ANCOVA with change scores as the dependent variable, treatment as the fixed group effect and pretreatment neurocognitive composite score as the covariate.

Independent two-way analyses of variance were used to test treatment effects on psychopathology measures, which were entered as the dependent variables, with treatment as the fixed group effect and time as the within-group effect. 
For all statistical tests, the level of significance was set at $P<0.05$.

\section{Results}

Baseline demographic and clinical characteristics are presented in Table 1. There was no significant difference between groups in demographic, clinical and cognitive variables, although the SoCIAL group showed higher education, MCCB composite score and FEIT total score.

\section{Treatment effect on the primary outcome measures}

ANCOVA showed no treatment effect on FEIT total score $(F=1.74, P=0.32)$ and a significant treatment effect on TASIT global score $(F=6.75, P \leq 0.04$, effect size $=0.32)$, as shown in Figure 2. Post hoc $t$-test for dependent samples on the TASIT global score showed a significant improvement in the SoCIAL group ( $t=3.31, P \leq 0.03$; Figure 2$)$ and no significant change in the SSANIT group $(t=-0.75, P=0.5)$.

\section{Treatment effects on the secondary outcome measures}

ANCOVA showed no significant treatment effect for the neurocognitive composite score $(F=0.002, P=0.96)$.

Two-way analyses of variance did not show a significant treatment effect for any psychopathological dimension ( $F$ from 0.19 to $6.38, P$ from 0.25 to 0.06 ).

A significant time effect was observed only in the SoCIAL group for the negative dimension "Avolition" ( $F=9.85, P \leq 0.04$; Figure 3).

Table I Baseline characteristics of the two groups

\begin{tabular}{|c|c|c|c|}
\hline & $\begin{array}{l}\text { SoCIAL } \\
(n=5)\end{array}$ & $\begin{array}{l}\text { SSANIT } \\
(n=5)\end{array}$ & $P$-value \\
\hline Age (years $\pm S D)$ & $36.4 \pm 13.1$ & $37.25 \pm 4.2$ & 0.9 \\
\hline Education (years, mean $\pm S D$ ) & $15.2 \pm 2.5$ & $11.5 \pm 2.4$ & 0.06 \\
\hline Duration of illness (years, mean \pm SD) & $6 \pm 2.4$ & $6 \pm 3.6$ & I \\
\hline PANSS positive (mean \pm SD) & $7.6 \pm 4.5$ & $1 I \pm 7.0$ & 0.4 \\
\hline PANSS disorganization (mean \pm SD) & $3 \pm 1.4$ & $4 \pm 2.3$ & 0.44 \\
\hline BNSS avolition (mean \pm SD) & $12.4 \pm 9.5$ & $18.25 \pm 6.4$ & 0.78 \\
\hline BNSS expressive deficit (mean \pm SD) & $6.8 \pm 10.3$ & $5.8 \pm 4.6$ & 0.88 \\
\hline UPSA-B (mean \pm SD) & $74.76 \pm 15.8$ & $65.73 \pm 5.5$ & 0.31 \\
\hline SLOF global score (mean \pm SD) & $99.6 \pm 23.8$ & $93 \pm 19.9$ & 0.71 \\
\hline TASIT (mean \pm SD) & $107.2 \pm 24.9$ & || $4 \pm 7.2 \mid$ & 0.58 \\
\hline FEIT (mean \pm SD) & $43.8 \pm 5.6$ & $29.8 \pm 15.4$ & 0.11 \\
\hline MCCB composite score (mean \pm SD) & $39.6 \pm 9.2$ & $29.6 \pm 9.4$ & 0.13 \\
\hline
\end{tabular}

Abbreviations: SoCIAL, Social Cognition Individualized Activities Lab; SSANIT, Social Skills And Neurocognitive Individualized Training; PANSS, Positive and Negative Syndrome Scale; BNSS, Brief Negative Symptom Scale; UPSA-B, University of California San Diego Performance-based Skills Assessment Brief; SLOF, Specific Level of Functioning Scale; TASIT, the Awareness of Social Inference Test; FEIT, Facial Emotion Identification Test; MCCB, MATRICS consensus cognitive battery.

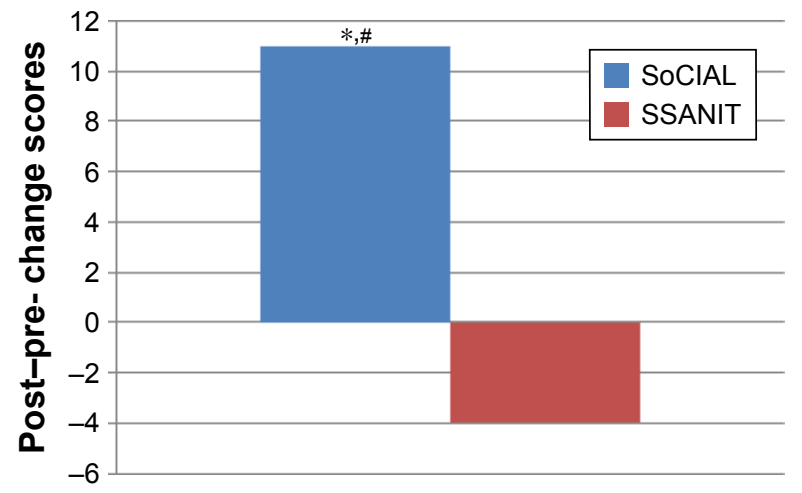

Figure 2 Treatment effect on TASIT global change scores.

Notes: *Significant treatment effect favoring the SoCIAL versus the SSANIT intervention, $P<0.04$; "significant within-group effect: improvement in the TASIT global score only in the SoCIAL group.

Abbreviations: TASIT, the Awareness of Social Inference Test; SoCIAL, Social Cognition Individualized Activities Lab; SSANIT, Social Skills And Neurocognitive Individualized Training.

ANCOVA showed no significant effect for functional capacity $(F=2.40, P=0.17)$ and real-life functioning (global SLOF score $F=0.89, P=0.38$ ).

\section{Patient's satisfaction}

The average score reported on CSQ was 28.6 for SoCIAL and 27.6 for SSANIT, indicating high satisfaction for both interventions.

\section{Discussion}

Our pilot study provides initial evidence for the feasibility and efficacy of a new social cognitive intervention for outpatients affected by schizophrenia. In a controlled trial

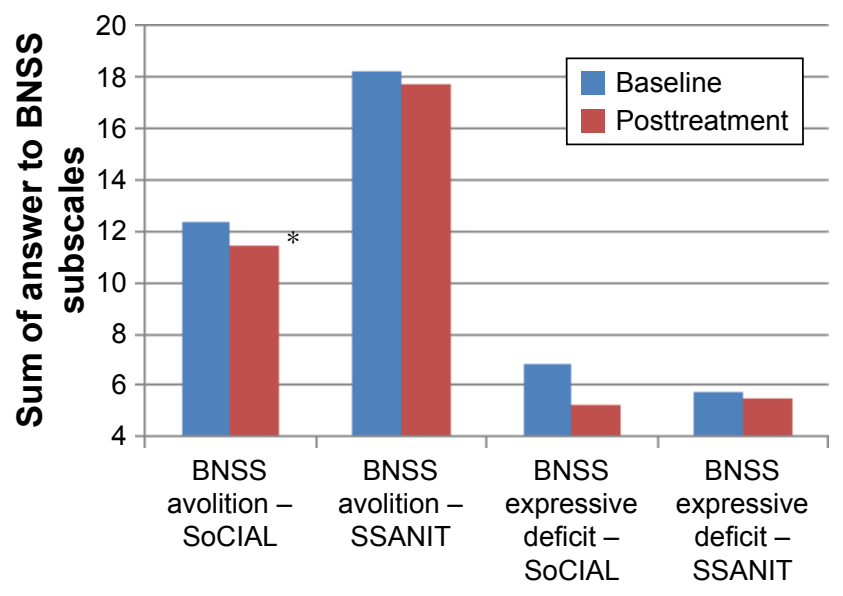

Figure 3 Time effect on the negative symptom domains.

Note: $* P<0.05$.

Abbreviations: BNSS, Brief Negative Symptom Scale; SoCIAL, Social Cognition Individualized Activities Lab; SSANIT, Social Skills And Neurocognitive Individualized Training. 
design, patients who received the SoCIAL intervention obtained significant improvements in SC after 20 weeks of treatment.

Although the groups receiving the two interventions were comparable at baseline for all variables, after 20 weeks of treatment, only patients assigned to SoCIAL had a significant improvement of SC. This result is even more significant when considering that both the interventions included an NIT. Our data suggest that the effect of the SoCIAL intervention on the SC domain was not due to an improvement of neurocognitive functions: if this was the case, the SoCIAL group should have presented a greater improvement on neurocognition; instead, it did not differ in change score with respect to the SSANIT group.

The design of this pilot study was aimed to verify whether a training specifically addressing ER and ToM had any advantage over a consolidated social skills training program when both the interventions were integrated with a neurocognitive training. The advantage of SoCIAL over SSANIT on SC suggests that this domain is amenable to remediation only using dedicated programs, specifically targeting its components.

Furthermore, only participants in the SoCIAL group showed an improvement of negative symptom domain avolition. This result is in line with evidences of a relationship between SC and negative symptoms. ${ }^{43-45}$ In a study conducted by Lincoln et $\mathrm{al},{ }^{45}$ the negative symptoms were significantly associated with deficits in ToM, explaining $26 \%$ of the variance.

No effect was observed for either intervention on positive symptoms, disorganization and functional capacity, in agreement with most of the literature findings. ${ }^{19,20,46}$

Finally, no effect was observed for either intervention on real-life functioning, despite the improvement of neurocognition in both groups and of SC and negative symptoms in the SoCIAL group. A reduced power to detect changes in functioning could be hypothesized, but the significance of effects on SC argues against this hypothesis. A more plausible hypothesis is that the lack of significant changes in functioning as measured by the SLOF might be related to the relatively high levels of functioning at baseline, with ceiling effects also on community activities, generally impaired in most of the community-dwelling patients. This hypothesis can explain the negative results observed with the SSANIT program, for which we had previously observed an impact on functioning. ${ }^{29}$

Some limitations of our pilot study should be discussed. Participants randomized to the SoCIAL group had a better ER at baseline, and this could be considered one of the reasons for their improvement after treatment. However, the baseline difference was not significant and no effect of treatment was observed for the FEIT change score which could be influenced more directly by the baseline difference on ER.

The main limitation of the study is the low statistical power due to the small sample size. It could be that we did not detect some treatment effects due to the small sample size. However, the two samples were comparably small, and even with a conservative ANCOVA design, we could demonstrate a significant treatment effect on the primary outcome. A sample extension will allow us to better understand the advantages of the SoCIAL versus the SSANIT.

In conclusion, the major results obtained in our pilot study are the improvement of SC and avolition only in the participants receiving SoCIAL that specifically addressed the SC domain. Our results are in line with a recent meta-analysis of SC remediation programs ${ }^{27}$ showing a moderate-large effect on $\mathrm{SC}$ dimensions, such as facial affect recognition and ToM, and a small to moderate effect on negative symptoms. The inclusion of both a neurocognitive and an SC remediation program might represent the most promising intervention with a potential impact on core dysfunctions of people with schizophrenia.

\section{Acknowledgments}

The authors wish to thank Dr Francesco Mancuso for his participation in the development of the SoCIAL intervention.

The development of SoCIAL was supported by an unrestricted grant from Otsuka Pharmaceutical Italy s.r.l., which had no role in the study design and conduction, data analysis, as well as in the manuscript preparation and submission.

\section{Disclosure}

The authors report no conflicts of interest in this work.

\section{References}

1. Galderisi S, Bucci P, Mucci A, et al. Categorical and dimensional approaches to negative symptoms of schizophrenia: focus on longterm stability and functional outcome. Schizophr Res. 2013;147(1): $157-162$.

2. Galderisi S, Davidson M, Kahn RS, et al; EUFEST group. Correlates of cognitive impairment in first episode schizophrenia: the EUFEST study. Schizophr Res. 2009;115(2-3):104-114.

3. Galderisi S, Maj M, Mucci A, et al. Historical, psychopathological, neurological, and neuropsychological aspects of deficit schizophrenia: a multicenter study. Am J Psychiatry. 2002;159(6):983-990.

4. Green MF, Harvey PD. Cognition in schizophrenia: past, present, and future. Schizophr Res Cogn. 2014;1(1):e1-e9.

5. Green MF, Kern RS, Braff DL, Mintz J. Neurocognitive deficits and functional outcome in schizophrenia: are we measuring the "right stuff"? Schizophr Bull. 2000;26(1):119-136.

6. Mucci A, Rucci P, Rocca P, et al. The specific level of functioning scale: construct validity, internal consistency and factor structure in a large Italian sample of people with schizophrenia living in the community. Schizophr Res. 2014;159(1):144-150. 
7. Schmidt SJ, Mueller DR, Roder V. Social cognition as a mediator variable between neurocognition and functional outcome in schizophrenia: empirical review and new results by structural equation modeling. Schizophr Bull. 2011;37(Supp1 2):S41-S54.

8. Karow A, Wittmann L, Schottle D, Schafer I, Lambert M. The assessment of quality of life in clinical practice in patients with schizophrenia. Dialogues Clin Neurosci. 2014;16(2):185-195.

9. Galderisi S, Rossi A, Rocca P, et al; Italian Network For Research on Psychoses. The influence of illness-related variables, personal resources and context-related factors on real-life functioning of people with schizophrenia. World Psychiatry. 2014;13(3):275-287.

10. Velligan DI, Mahurin RK, Diamond PL, Hazleton BC, Eckert SL, Miller AL. The functional significance of symptomatology and cognitive function in schizophrenia. Schizophr Res. 1997;25(1):21-31.

11. Galderisi S, Rossi A, Rocca P, et al; Italian Network for Research on Psychoses. Pathways to functional outcome in subjects with schizophrenia living in the community and their unaffected first-degree relatives. Schizophr Res. 2016;175(1-3):154-160.

12. Green MF, Leitman DI. Social cognition in schizophrenia. Schizophr Bull. 2008;34(4):670-672.

13. Harvey PD, Penn D. Social cognition: the key factor predicting social outcome in people with schizophrenia? Psychiatry (Edgmont). 2010; 7(2):41-44.

14. Couture SM, Penn DL, Roberts DL. The functional significance of social cognition in schizophrenia: a review. Schizophr Bull. 2006; 32(Suppl 1):S44-S63.

15. Fett AK, Viechtbauer W, Dominguez MD, Penn DL, van Os J, Krabbendam L. The relationship between neurocognition and socia cognition with functional outcomes in schizophrenia: a meta-analysis Neurosci Biobehav Rev. 2011;35(3):573-588.

16. Mancuso F, Horan WP, Kern RS, Green MF. Social cognition in psychosis: multidimensional structure, clinical correlates, and relationship with functional outcome. Schizophr Res. 2011;125(2-3):143-151.

17. Davidson M, Galderisi S, Weiser M, et al. Cognitive effects of antipsychotic drugs in first-episode schizophrenia and schizophreniform disorder: a randomized, open-label clinical trial (EUFEST). Am J Psychiatry. 2009;166(6):675-682.

18. Hill SK, Bishop JR, Palumbo D, Sweeney JA. Effect of secondgeneration antipsychotics on cognition: current issues and future challenges. Expert Rev Neurother. 2010;10(1):43-57.

19. Wykes T, Reeder C, Landau S, et al. Cognitive remediation therapy in schizophrenia: randomised controlled trial. Br J Psychiatry. 2007;190: 421-427.

20. Wykes T, Huddy V, Cellard C, McGurk SR, Czobor P. A meta-analysis of cognitive remediation for schizophrenia: methodology and effect sizes. Am J Psychiatry. 2011;168(5):472-485.

21. Penn DL, Roberts DL, Combs D, Sterne A. Best practices: the development of the Social Cognition and Interaction Training program for schizophrenia spectrum disorders. Psychiatr Serv. 2007;58(4):449-451.

22. Wolwer W, Frommann N. Social-cognitive remediation in schizophrenia: generalization of effects of the Training of Affect Recognition (TAR). Schizophr Bull. 2011;37(Suppl 2):S63-S70.

23. Horan WP, Kern RS, Shokat-Fadai K, Sergi MJ, Wynn JK, Green MF. Social cognitive skills training in schizophrenia: an initial efficacy study of stabilized outpatients. Schizophr Res. 2009;107(1):47-54.

24. Combs DR, Tosheva A, Penn DL, Basso MR, Wanner JL, Laib K. Attentional-shaping as a means to improve emotion perception deficits in schizophrenia. Schizophr Res. 2008;105(1-3):68-77.

25. McGurk SR, Twamley EW, Sitzer DI, McHugo GJ, Mueser KT. A metaanalysis of cognitive remediation in schizophrenia. Am J Psychiatry. 2007;164(12):1791-1802.

26. Roberts DL, Penn DL. Social cognition and interaction training (SCIT) for outpatients with schizophrenia: a preliminary study. Psychiatry Res. 2009;166(2-3):141-147.

27. Kurtz MM, Richardson CL. Social cognitive training for schizophrenia: a meta-analytic investigation of controlled research. Schizophr Bull. 2012;38(5):1092-1104.
28. Barlati S, Deste G, De Peri L, Ariu C, Vita A. Cognitive remediation in schizophrenia: current status and future perspectives. Schizophr Res Treatment. 2013;2013:156084.

29. Galderisi S, Piegari G, Mucci A, et al. Social skills and neurocognitive individualized training in schizophrenia: comparison with structured leisure activities. Eur Arch Psychiatry Clin Neurosci. 2010;260(4):305-315.

30. Bucci P, Piegari G, Mucci A, et al. Neurocognitive individualized training versus social skills individualized training: a randomized trial in patients with schizophrenia. Schizophr Res. 2013;150(1):69-75.

31. Larsen DL, Attkisson CC, Hargreaves WA, Nguyen TD. Assessment of client/patient satisfaction: development of a general scale. Eval Program Plann. 1979;2(3):197-207.

32. Wallwork RS, Fortgang R, Hashimoto R, Weinberger DR, Dickinson D. Searching for a consensus five-factor model of the Positive and Negative Syndrome Scale for schizophrenia. Schizophr Res. 2012; 137(1-3):246-250.

33. Mucci A, Galderisi S, Merlotti E, et al; Italian Network for Research on Psychoses. The Brief Negative Symptom Scale (BNSS): Independent validation in a large sample of Italian patients with schizophrenia. Eur Psychiatry. 2015;30(5):641-647.

34. Strauss GP, Keller WR, Buchanan RW, et al. Next-generation negative symptom assessment for clinical trials: validation of the Brief Negative Symptom Scale. Schizophr Res. 2012;142(1-3):88-92.

35. Kern RS, Nuechterlein KH, Green MF, et al. The MATRICS Consensus Cognitive Battery, part 2: co-norming and standardization. Am J Psychiatry. 2008;165(2):214-220.

36. Nuechterlein KH, Green MF, Kern RS, et al. The MATRICS Consensus Cognitive Battery, part 1: test selection, reliability, and validity. Am J Psychiatry. 2008;165(2):203-213.

37. Kerr SL, Neale JM. Emotion perception in schizophrenia: specific deficit or further evidence of generalized poor performance? J Abnorm Psychol. 1993;102(2):312-318.

38. McDonald S, Bornhofen C, Shum D, Long E, Saunders C, Neulinger K. Reliability and validity of The Awareness of Social Inference Test (TASIT): a clinical test of social perception. Disabil Rehabil. 2006;28(24): 1529-1542.

39. Mausbach BT, Harvey PD, Goldman SR, Jeste DV, Patterson TL. Development of a brief scale of everyday functioning in persons with serious mental illness. Schizophr Bull. 2007;33(6):1364-1372.

40. Sabbag S, Twamley EW, Vella L, Heaton RK, Patterson TL, Harvey PD. Predictors of the accuracy of self assessment of everyday functioning in people with schizophrenia. Schizophr Res. 2012;137(1-3):190-195.

41. Benton MK, Schroeder HE. Social skills training with schizophrenics: a meta-analytic evaluation. J Consult Clin Psychol. 1990;58(6): 741-747.

42. Bellack AS, Sayers M, Mueser KT, Bennett M. Evaluation of social problem solving in schizophrenia. J Abnorm Psychol. 1994;103(2): 371-378.

43. Kalin M, Kaplan S, Gould F, Pinkham AE, Penn DL, Harvey PD. Social cognition, social competence, negative symptoms and social outcomes: inter-relationships in people with schizophrenia. J Psychiatr Res. 2015;68:254-260.

44. Lin CH, Huang CL, Chang YC, et al. Clinical symptoms, mainly negative symptoms, mediate the influence of neurocognition and social cognition on functional outcome of schizophrenia. Schizophr Res. 2013; 146(1-3):231-237.

45. Lincoln TM, Mehl S, Kesting ML, Rief W. Negative symptoms and social cognition: identifying targets for psychological interventions. Schizophr Bull. 2011;37(Suppl 2):S23-S32.

46. Wykes T, Spaulding WD. Thinking about the future cognitive remediation therapy - what works and could we do better? Schizophr Bull. 2011;37(Suppl 2):S80-S90.

47. Kay SR, Fiszbein A, Opler LA. The positive and negative syndrome scale (PANSS) for schizophrenia. Schizophr Bull. 1987;13(2):261-276. 


\section{Publish your work in this journal}

Neuropsychiatric Disease and Treatment is an international, peerreviewed journal of clinical therapeutics and pharmacology focusing on concise rapid reporting of clinical or pre-clinical studies on a range of neuropsychiatric and neurological disorders. This journal is indexed on PubMed Central, the 'PsycINFO' database and CAS,

and is the official journal of The International Neuropsychiatric Association (INA). The manuscript management system is completely online and includes a very quick and fair peer-review system, which is all easy to use. Visit http://www.dovepress.com/testimonials.php to read real quotes from published authors.

Submit your manuscript here: http://www.dovepress.com/neuropsychiatric-disease-and-treatment-journal 